\title{
Comparación de métodos basados en los requerimientos nutricionales y disponibilidad de biomasa para estimar la capacidad de carga para venado cola blanca
}

\author{
Comparison of methods based on the nutritional requirements and availability \\ of biomass to estimate carrying capacity for white tailed deer \\ FX Plata ${ }^{a *}$, GD Mendoza $^{\mathrm{a}}$, JA Viccon ${ }^{\mathrm{a}}$, R Bárcena ${ }^{\mathrm{b}}$, F Clemente $^{\mathrm{b}}$ \\ aUniversidad Autónoma Metropolitana, Doctorado en Ciencias Biológicas, México D.F. \\ belegio de Postgraduados, Campus Montecillo, México.
}

\begin{abstract}
SUMMARY
Due to the importance of the estimation of carrying capacity ( $K$ animals/ha) in white tailed deer, the objectives of this study were: 1$)$ To compare the estimation of $K$ with five methods, three of them based on nutrient availability (dry matter (DM), digestible energy (DE) and nitrogen (N)), one based on pressure of pasture and one based on the requirement of metabolizable energy (ME) based on the ecological metabolism of the deer, and;2) To compare the $K$ estimated when using the total biomass or vegetation groups consumed by the deer (grasses, herbs, shrubs and trees). The information about diet composition and biomass was collected at a hunting ranch in Aguascalientes, Mexico. The results were analyzed as a factorial arrangement $(5 \mathrm{x} 4)$, with the factors being the estimation method and the vegetal group factors. The estimated $K$ (deer/ha) with total biomass was the highest (1.043), followed by grass $(0.707)$ and herbs $(0.325)$, whereas the estimates with trees and shrubs were the lowest ones $(0.228$ and 0.032 deer/ha). The $K$ estimated, based in shrubs availability gives the value most similar to the population density than with the other methods, independently of selected method (DM, DE or $\mathrm{N}$ availability, pressure of pasture or ecological metabolism). It is concluded that carrying capacity should be estimated based on shrubs availability when considering the population density ( $0.02-0.04$ deer/ha) as the appropriate indicator to evaluate the carrying capacity.
\end{abstract}

Palabras clave: capacidad de carga, Odocoileus virginianus, requerimientos nutricionales, vegetación.

Key words: carrying capacity, Odocoileus virginianus, nutritional requirements, vegetation.

\section{INTRODUCCIÓN}

La estimación del número de animales que un hábitat puede mantener en una condición vigorosa y saludable es un problema que tiene sus orígenes a comienzos del siglo pasado. Por tal razón y a lo largo de todo este tiempo se han generado y discutido diversos tipos de modelos que permiten su determinación (McLeod 1997). Dependiendo de la especie y función para la que sea utilizada, la capacidad de carga ha sido definida de diversas maneras (Miller y Wentworth 2000). En general, la capacidad de carga ecológica $(K)$ es definida como el número máximo de animales que puede crecer dentro de una población de acuerdo al modelo logístico, mientras que las restricciones a dicho crecimiento incluyen la disponibilidad de espacio, agua y biomasa comestible, de tal forma que la tasa de crecimiento $(r)$ es igual a cero cuando una población alcanza $K$ (Gotelli 2008). Esta $K$ es afectada por la presencia de enfermedades que afectan a la población, la disponibilidad de agua, la presencia de competidores naturales y depredadores (Gallina 1990, Mandujano 2007). Por otro lado, la capacidad de carga económica se refiere

Aceptado: 15.09.2010.

* Calz. Del hueso 1100, México D.F. 04960, México; ppfx221@ correo.xoc.uam.mx al valor que permite maximizar el número de individuos a condición de no generar cambios en la composición botánica del sitio (Beck y col 2006). Mientras que para los productores de ganado doméstico y los manejadores de pastizales, la estimación de la carga animal representa al número máximo de animales en un pastizal que permite el mantenimiento o mejoramiento de la vegetación o de los recursos utilizados, y se utiliza como sinónimo de $K$ (Jacoby 1989, Young 1998, Galt 2000). En todos los casos, la $K$ de una región, es dependiente del tipo y disponibilidad de vegetación presente, de los hábitos de consumo de la especie y la época del año (Stuth y Sheffield 2001).

Se han evaluado diferentes métodos para determinar la capacidad de carga; Mandujano (2007) evaluó la capacidad de carga de un área del estado de Jalisco (México) utilizando el concepto ecológico y considerando que $K$ es igual a la densidad estimada de animales más los animales muertos en el área. En bovinos, Paladines y Lazcano (1983) diseñaron una técnica para estimarla incorporando el concepto de presión de pastoreo (PP), el cual en lugar de estimar el consumo del animal asigna un porcentaje de su peso vivo como requerimiento de forraje. En venados, Hobbs y Swift (1985), McCall y col (1997) y Stuth y Sheffield (2001), han evaluado métodos que utilizan la materia seca o los nutrientes disponibles en el área y los dividen entre los requerimientos nutritivos. Sin embargo, dichos requerimientos 
son definidos en forma fija (Robbins 1973, McCall y col 1997, Galbraith y col 1998), lo que fisiológicamente no ocurre en los animales. Debido a que los requerimientos nutricionales cambian con la época del año, el género y el estado fisiológico del animal, Moen (1978) desarrolló un modelo para estimar el gasto energético de un venado cola blanca en función de estos factores y definió a este requerimiento de energía como metabolismo ecológico del venado $(M E V)$; este modelo fue modificado por Clemente (1984), para estimar el $M E V$ como requerimiento de energía metabolizable (EM) en función del peso vivo.

La estimación adecuada de $K$ es fundamental para garantizar tanto el mantenimiento de la población de venados como el de la comunidad vegetal. Sin embargo, uno de los problemas que se tienen al determinar la $K$ de hábitats para venado cola blanca en vida libre es que algunos métodos de estimación utilizan procedimientos similares a los usados con bovinos domésticos sin considerar su selectividad (McCall y col 1997, Stuth y Sheffield 2001). Los hábitos alimenticios del venado son de ramoneo y el tipo de vegetales que consumen son casi totalmente diferentes a los consumidos por los bovinos. Se ha demostrado que aun bajo condiciones de excelente pastizal, sólo consumen alrededor de un $8 \%$ de gramíneas del total de su dieta (Hansen y col 1977, Stuth y Winward 1977, Bryant y col 1979). Otros autores han mostrado que la palatabilidad de las arbustivas y las herbáceas es un factor determinante en la selección de la dieta de venados y que pueden incluso minimizar el consumo de leguminosas o gramíneas si este tipo de arbustivas están presentes (Augustine y Jordan 1998, Sauvé y Côté 2007, Plata y col 2009). También se ha demostrado que el uso de hábitat por esta especie es dependiente del tipo de especies vegetales que lo conforman, de tal manera que aunque un área ofrezca mayor biomasa vegetal comestible los venados pueden localizarse en mayor densidad en otras áreas con menor densidad relativa de alimento (Mandujano y col 2004).

Considerando que el metabolismo energético del venado cola blanca se modifica en una forma dinámica y que el modelo de presión de pastoreo utilizado en bovinos no ha sido evaluado en venado cola blanca, los objetivos de este trabajo fueron: 1) Comparar la estimación de $K$ por cinco métodos, tres basados en disponibilidad de componentes nutritivos: materia seca (MS), energía digestible (ED), nitrógeno (N), otro basado en la presión de pastoreo y un último en el requerimiento de EM estimado con base al $M E V$, y 2) Estimar los cambios en la $K$ al sustituir en los modelos evaluados la biomasa total por los grupos de vegetación consumidos por el venado (arbóreas, herbáceas, arbustivas y gramíneas) comparando los resultados de las estimaciones de capacidad de carga versus la densidad poblacional de venados reportada de la zona.

\section{MATERIAL Y MÉTODOS}

\section{SITIO DE MUESTREO}

Se usó la información del muestreo de vegetación realizado por Clemente (1984) en un rancho cinegético ("El Antrialgo") localizado en el municipio de San José de Gracia, Aguascalientes, México, entre los paralelos $22^{\circ} 05^{\prime}$ y $22^{\circ} 10^{\prime}$ latitud $\mathrm{N}$ y los $102^{\circ} 35^{\prime}$ y $102^{\circ} 40^{\prime}$ latitud $\mathrm{W}$, dentro de la zona sujeta a conservación ecológica denominada "Sierra Fría". El rancho tiene una extensión de 260 ha, con una altura sobre el nivel del mar de 2.350 msnm. La precipitación anual de la región fue de $600 \mathrm{~mm}$ distribuidos principalmente de junio a octubre, la evaporación media anual es de $1.500 \mathrm{~mm}$, y el clima corresponde al tipo BSwh de acuerdo a la clasificación de Koppen, modificada por García (1981). El tipo de suelo del área de estudio va de suelo complejo de montaña podzólico a suelo de depósito abanico aluvial y lacustre. La relación de especies que componen la cubierta vegetal del lugar se observa en el cuadro 1. Este rancho se caracterizó por tener actividad ganadera cuatro años antes de convertirse en rancho cinegético.

\section{MUESTREO DE VEGETACIÓN}

El muestreo de vegetación se realizó en los meses de julio (antes de la lluvia), octubre (época de lluvias) y diciembre (después de las lluvias) de 1983. Dichos muestreos corresponden a los momentos más importantes de cambios vegetativos (Beauchamp y Stromberg 2008, Rogers y col 2004) y no se muestreó al inicio del año por considerar que al no haber precipitación pluvial los cambios en crecimiento eran nulos (Otto y col 2002).

Tomando como criterio la dominancia de especies arbóreas y arbustivas, se determinaron tres tipos de hábitats. El primero dominado por Quercus-Arctostaphylus, el segundo dominado por Quercus-Arctostaphylus-Juniperus, mientras que el tercero por Quercus-Juniperus. Todos los muestreos se realizaron en el año de 1984. Para el muestreo de julio se localizaron tres áreas representativas de cada hábitat y para los muestreos de octubre y diciembre se localizaron tres áreas representativas del primero y un área representativa del segundo y el tercero. En total se tuvieron 19 sitios de muestreo de $400 \mathrm{~m}^{2}$ (20 x $20 \mathrm{~m}$ ) cada uno.

\section{ESTIMACIÓN DE BIOMASA}

Para las especies arbóreas de cada árbol dentro del área de muestreo se recolectó el material vegetativo disponible a 1,60 m de altura (Jordan 1967). Para las arbustivas menores a esa altura se colectó la biomasa total separando las hojas tiernas, rebrotes y frutos. Para las determinaciones de la disponibilidad de forraje (herbáceas y gramíneas) dentro de cada área de muestreo se distribuyeron al azar 2 cuadrantes de $1 \mathrm{~m}^{2}$ y se recolectó todo el material herbáceo y 
Cuadro 1. Especies que componen la cubierta vegetal del rancho El Antrialgo.

Vegetal species at the soil cover at the Antrialgo Ranch.

\begin{tabular}{|c|c|c|}
\hline Arctostaphylos pungens & Ericaceae & Arbustiva \\
\hline Aristida orcuttiana & Poaceae & Gramínea \\
\hline Borreria densiflora & Rubiaceae & Herbácea \\
\hline Carex coulteri & Cyperaceae & Herbácea \\
\hline Calea spp & Asteraceae & Herbácea \\
\hline Castilleja tenuiflora & Scrophulariaceae & Arbustiva \\
\hline Cologania angustifolia & Fabaceae & Herbácea \\
\hline Cologania obovata & Fabaceae & Herbácea \\
\hline Conyza gnaphalioides & Asteraceae & Herbácea \\
\hline Cyperus seslerioides & Cyperaceae & Herbácea \\
\hline Evolvulus prostratus & Convolvulaceae & Herbácea \\
\hline Geranium spp & Geraniaceae & Arbustiva \\
\hline Allium glomeratum & Liliaceae & Herbácea \\
\hline Hypericum silenoides & Clusiaceae & Herbácea \\
\hline Ipomoea capillacea & Convolvulaceae & Herbácea \\
\hline Juniperus deppeana & Cupressaceae & Arbórea \\
\hline Lycurus phleoides & Poaceae & Gramínea \\
\hline Muhlenbergia emersleyi & Poaceae & Gramínea \\
\hline Muhlenbergia pungens & Poaceae & Gramínea \\
\hline Muhlenbergia rigida & Poaceae & Gramínea \\
\hline Odontotrichum amplum & Asteraceae & Herbácea \\
\hline Oxalis latifolia & Oxalidaceae & Herbácea \\
\hline Perymenium buphthalmoides & Asteraceae & Arbustiva \\
\hline Polygala alba & Polygalaceae & Herbácea \\
\hline Quercus rugosa & Fagaceae & Arbustiva \\
\hline Quercus microphylla & Fagaceae & Arbustiva \\
\hline Stevia pilosa & Asteraceae & Herbácea \\
\hline Stevia serrata & Asteraceae & Herbácea \\
\hline Stipa virescens & Poaceae & Gramínea \\
\hline Viola barroetana & Violaceae & Herbácea \\
\hline
\end{tabular}

gramíneo presente en el suelo (Bullock 1996). La biomasa vegetal de cada estrato se estimó como el producto del área total por el promedio de los kg de MS del mismo en ese periodo, mientras que la biomasa total fue el resultado de la sumatoria de las biomasas de todos los estratos en el mismo periodo.

\section{COMPOSICIÓN QUÍMICA DE LA BIOMASA VEGETAL}

Tanto las especies analizadas como las características nutritivas, la composición química y la digestibilidad in vitro de la dieta de acuerdo a cada época fueron reportadas previamente (Clemente y col 2005). La concentración de energía bruta del alimento se determinó de acuerdo a los métodos oficiales de AOAC (1995). La concentración de energía digestible (ED) de cada estrato vegetal se estimó con el producto de la relación EDMS Mcal/kg = $($ DIVMS/100) $\times$ EB utilizando la digestibilidad in vitro de los diferentes grupos vegetales en cada época del año y la relación de energía metabolizable se estimó con la ecuación $\mathrm{EM}=0,82 \times \mathrm{ED}$ (González y Everitt 1982) .

\section{REQUERIMIENTOS NUTRITIVOS DEL ANIMAL}

Para la estimación del consumo de materia seca (CMS) y los requerimientos de $\mathrm{ED}$ y $\mathrm{N}$, se consideró un venado de $60 \mathrm{~kg}$ de peso vivo (PV). El CMS en estos modelos se estimó con el valor obtenido por Galbraith y col (1998) de $46 \mathrm{~g}$ de MS por kilogramo de peso metabólico (CMS kg/ día $=0,046 * \mathrm{PV}^{0,75}$ ). La estimación del requerimiento de $\mathrm{ED}$ (RED) para mantenimiento fue de $158 \mathrm{kcal}$ por kilogramo de peso metabólico $\left(\mathrm{RED}=158 * \mathrm{PV}^{0,75}\right.$; Ullrey y col 1970). El requerimiento de nitrógeno $(\mathrm{RN})$ se estimó en $710 \mathrm{mg}$ por kilogramo de peso metabólico $\left(\mathrm{RN}\right.$ g/día $=0,710 * \mathrm{PV}^{0,75}$; Robbins 1973). El metabolismo ecológico del venado (MEV, kcal/día) se calculó utilizando una hoja de cálculo del programa 
Excel de Microsoft Office y se encuentra disponible en la plataforma de Bioeficiencia ${ }^{1}$ que corresponde al modelo de Moen (1978) modificado por Clemente (1984), con el cual se estimó el requerimiento energético considerando los días julianos 31, 59, 90, 120, 151, 181, 212, 243, 273, 304, 330 y 365 que corresponden al día último de cada mes, respectivamente, y se multiplicó por el número de días de cada mes.

\section{DENSIDAD ANIMAL}

La densidad poblacional de venado en el año durante el cual se hizo el muestreo de vegetación en la zona fue reportada por Romo (1987), quien estimó la densidad de venados en la zona aplicando dos técnicas diferentes: un muestreo por transectos de observación directa y un muestreo por conteo indirecto basado en indicios (huellas). También se consideraron los antecedentes publicados por Villalobos (1998), quien reportó estimados de la población de venados en Sierra Fría desde 1975 hasta 1996. Estas estimaciones fueron realizadas a partir de 24 muestreos realizados en un área de 2.975 ha. Ambas densidades junto con las de Kobelkowsky y col (2000) fueron tomadas como indicadores de referencia para discutir la estimación de la capacidad de carga (Mandujano 2007).

\section{ESTIMACIÓN DE CAPACIDAD DE CARGA}

Se estimó la $K$ del área muestreada utilizando los modelos descritos a continuación. En todos los métodos la eficiencia de utilización fue del $35 \%$, ya que en el modelo logístico de crecimiento dicha asignación garantiza que el crecimiento de una población no se limita (Gotelli 1998). La estimación se realizó para cada periodo de muestreo considerando su duración (91 días el verano, 61 días el otoño y 213 días el período de invierno).

\section{MODELOS EVALUADOS}

\section{MODELOS DE DISPONIBILIDAD DE NUTRIENTES}

Se seleccionaron tres de los métodos publicados por McCall y col (1997) basados en la disponibilidad de MS, ED y N. Para estimar la $K$ con la biomasa total se usó el algoritmo de Hobbs y Swift (1985), utilizando la ecuación 1. Para la estimación de la $K$ por grupo vegetal se consideró la concentración del nutriente evaluado (MS, ED o N) en cada uno de los estratos vegetales presentes en el área, utilizando la ecuación 2.

$$
K=0,35\left(\frac{\sum_{i=1}^{n} B i * F j}{R D}\right)
$$

1 Modelación sobre eficiencia animal. http://bioeficiencia.avanzavet. com/ consultada el 12 de febrero de 2008 .

$$
K=0,35\left(\frac{B i * F j}{R D}\right)
$$

Donde:

$K=$ Carga del área de estudio (venados/ha).

$B i=$ Biomasa del estrato vegetal $i$ (gramíneas, herbáceas, arbustivas y arbóreas; $\mathrm{kg} / \mathrm{ha}$ ).

$F j=$ Contenido de los componentes nutritivos del estrato vegetal $j$ (MS kg, $\mathrm{N} \mathrm{kg/kg} \mathrm{o} \mathrm{ED} \mathrm{Mcal/kg).}$

$R=$ Requerimiento de los componentes nutritivos de un venado de $60 \mathrm{~kg}$ (kg o kcal).

$D=$ Tiempo de utilización (días).

0,35 = Eficiencia de utilización del forraje.

\section{MÉTODO BASADO EN LA PRESIÓN DE PASTOREO}

Para estimar la capacidad de carga con el método basado en la presión de pastoreo (Paladines y Lazcano 1983) se estimó la disponibilidad (D) de MS total o de cada estrato vegetal por ha y se aplicó la siguiente ecuación:

$$
K=\left(\frac{\left(\frac{D * 0,35 * A * 100}{T * P P}\right)}{60}\right)
$$

Donde:

$K=$ Capacidad de carga (venado/ha)

$\mathrm{D}=$ Disponibilidad de MS total o por estrato vegetal $(\mathrm{kg} / \mathrm{ha})$ 0,35 = Eficiencia de utilización del forraje

$\mathrm{A}=$ Área (ha)

$\mathrm{T}=$ Tiempo (días)

$\mathrm{PP}=$ Presión de pastoreo $(\%$ de $\mathrm{PV})$

60 = Peso vivo del venado $(\mathrm{kg})$

La presión de pastoreo asignada fue igual al factor de consumo proporcional al peso vivo (PV) propuesto por Stuth y Sheffield (2001) de 3,5\% del PV, el tiempo (T) cambió de acuerdo a la época del año. Para determinar el número de animales por ha $(K)$ que se pueden sostener con los diversos estratos vegetales se dividió el número de $\mathrm{kg}$ de animal entre 60.

En las metodologías basadas en la disponibilidad de MS, ED y N y en la presión de pastoreo, el consumo y los requerimientos fueron mantenidos constantes a lo largo del año.

\section{MÉTODO BASADO EN EL METABOLISMO ECOLÓGICO}

Este método se basó en una estimación del requerimiento energético del venado en vida libre, por una serie de ecuaciones que conforman el metabolismo ecológico (ME, kcal/día), el cual se calculó utilizando la secuencia 
descrita por Moen (1978) modificada por Clemente (1984). A diferencia de los métodos anteriores, el modelo de metabolismo ecológico es un método dinámico que cambia el requerimiento energético de acuerdo a la época del año junto con los cambios de actividad del venado. Para estimar la capacidad de carga en este modelo se dividió la energía metabolizable disponible de la biomasa total o de los diversos estratos vegetales entre la sumatoria del requerimiento energético del animal de los meses que corresponden a cada periodo, utilizando la siguiente fórmula:

$$
K=\frac{\sum_{1-n} E m f * A * 0,35}{\sum_{1-m} M E V_{d}}
$$

Donde:

$K=$ Capacidad de carga (venados de $60 \mathrm{~kg} / \mathrm{ha}$ )

$E m f$ = Energía metabolizable del forraje de la especie vegetal 1 hasta $\mathrm{n}(\mathrm{kcal} / \mathrm{ha})$

$A=$ Área total del predio (ha)

$n=$ Estrato

$m$ = Día juliano

$M E V_{d}=$ Metabolismo ecológico diario de un venado de $60 \mathrm{~kg}$ (kcal/día). El cálculo de esta variable se observa en el cuadro 2.

0,35 = Eficiencia de utilización del forraje

\section{ANÁLISIS ESTADÍSTICO}

Tanto el análisis estadístico de la biomasa de los diferentes grupos vegetales como de la capacidad de carga se realizaron utilizando un diseño en bloques al azar considerando a la época del año como factor de heterogeneidad y se realizó una prueba de Tukey para comparar las medias de los tratamientos, para lo cual se utilizó el procedimiento GLM del SAS (1994, SAS Inst. Inc. Cary, NC, USA). La biomasa total no se incluyó en dicho análisis por ser el resultado de la sumatoria de los grupos vegetales; en consecuencia, es mayor a cada uno de los mismos.

Los estimados de capacidad de carga se analizaron con un diseño de tratamientos con arreglo factorial (cinco por cinco), donde los factores fueron los métodos y los estratos vegetales consumidos por el venado (MS total, gramíneas, herbáceas, arbustivas y arbóreas). Por esto también se realizaron contrastes de interés, para comparar algunos efectos específicos tanto entre grupos vegetales como entre métodos (Herrera y Barreras 2005).

\section{RESULTADOS}

En el cuadro 1 se presenta la relación de las especies que componían la cubierta vegetal y que fueron muestreadas para la estimación de biomasa total, disponible y nutrientes aportados para la estimación de $K$. El análisis estadístico mostró que existieron diferencias estadísticas $(\mathrm{P}<0,05)$ tanto entre la biomasa vegetal total y disponible por grupo vegetal como en la concentración de energía digestible y metabolizable de los mismos (cuadro 3). Las gramíneas fueron el grupo que aportó la mayor cantidad de biomasa, energía digestible y metabolizable, mientras que las arbóreas y herbáceas aportaron cantidades intermedias y las arbustivas fueron las que menor cantidad de compuestos nutritivos aportaron. Sin embargo, la cantidad de $\mathrm{N}$ disponible por grupo vegetal no mostró cambios importantes $(\mathrm{P}>0,05)$. La época del año no modificó ni la biomasa total ni la disponibilidad de MS en cada estrato vegetal, ni tampoco tuvo efecto en los compuestos nutritivos disponibles. Debido a que no se encontraron efectos de la época del año, en el cuadro 3 se presentan las medias y el error estándar de la biomasa disponible y los compuestos nutritivos contenidos en cada estrato. Además, se presenta como referencia la biomasa total, biomasa disponible y compuestos nutritivos resultantes de la sumatoria de los grupos vegetales y los requerimientos de MS, ED, N y EM de un animal de $60 \mathrm{~kg}$ de peso vivo.

En el análisis estadístico de $K$ (cuadro 4) se encontraron diferencias significativas tanto entre los métodos de estimación $(\mathrm{P}<0,003)$ como entre la $K$ estimada a partir de los diversos grupos vegetales $(\mathrm{P}<0,0001)$; sin embargo, la interacción entre los mismos no fue significativa $(\mathrm{P}=0,20)$, por lo que sólo se presentan los efectos principales de método de estimación y grupo vegetal, los cuales se contrastan con la densidad poblacional

Cuadro 2. Modelos para estimar el metabolismo ecológico de los venados de cola blanca1.

Models for estimation of ecological metabolism of white-tailed deer.

Machos

$M E_{m}=[\{1,02850 \sin [(D j)(0,09863)+239]+0,0281\}+2,67] * 70\left\{\left[\left(e^{1,617+0,3810 \text { In edias }}\right) *\{\sin [D j)(0,9863)+90] * 0\right.\right.$

Hembras

$M E_{H}=2,56894 e^{0,19602(c)}\left(\left\{\left[1,0285 * \sin \left[\left(d j+170,0536 e^{0,15488(c)}\right) * 0,9863\right]+0,0281\right][0,19+0,05(c)]\right\}+[0,81-0,05(c)]\right) * 70$

$\left\{\left[\left(e^{1,617+0,3810 \text { In edias }}\right) *\{\sin [(D j)(0,98)\right.\right.$

1 La solución a la ecuación está disponible en Bioeficiencia. Modelación sobre eficiencia animal. http://bioeficiencia.avanzavet.com/ consultada el 12 de febrero de 2008. 
Cuadro 3. Biomasa, compuestos nutritivos disponibles por grupo vegetal y requerimientos del venado de cola blanca utilizados para estimar capacidad de carga con diferentes métodos.

Biomass, nutrient availability by vegetative group and white-tailed deer requirements used to estimate carrying capacity with different methods.

Aportes nutritivos

Biomasa disponible por grupo vegetal

\begin{tabular}{|c|c|c|c|c|c|c|}
\hline & Gramíneas & Herbáceas & Arbóreas & Arbustivas & Total & EEM \\
\hline Materia seca disponible (kg/ha) & $127,4^{\mathrm{a}}$ & $49,5^{\mathrm{ab}}$ & $38,3^{\mathrm{ab}}$ & $5,8^{\mathrm{b}}$ & 221,1 & 24,2 \\
\hline Proporción de la biomasa aportada (\%) & 57,3 & 22,4 & 17,3 & 2,6 & 100 & \\
\hline $\mathrm{ED}^{1}$ (Mcal/ha) & $135,4^{\mathrm{a}}$ & $113,4^{\mathrm{ab}}$ & $54,0^{\mathrm{ab}}$ & $\mathrm{a} 9,6^{\mathrm{b}}$ & 312,4 & 26,9 \\
\hline Proporción de la ED aportada (\%) & 43,3 & 36,4 & 17,3 & 3,1 & & \\
\hline $\mathrm{EM}^{2}(\mathrm{kcal} / \mathrm{ha})$ & $111,0^{\mathrm{a}}$ & $93,0^{\mathrm{ab}}$ & $44,3^{\mathrm{ab}}$ & $7,9^{\mathrm{b}}$ & 256,2 & 22,0 \\
\hline Proporción de la EM aportada (\%) & 43,3 & 36,3 & 17,3 & 3,1 & & \\
\hline Nitrógeno (kg/ha) & $0,76^{\mathrm{a}}$ & $0,64^{\mathrm{a}}$ & $0,38^{\mathrm{a}}$ & $0,05^{\mathrm{a}}$ & 1,83 & 1,0 \\
\hline Proporción del $\mathrm{N}$ aportado (\%) & 22,7 & 35,0 & 20,6 & 2,9 & & \\
\hline
\end{tabular}

Requerimientos anuales del venado de cola blanca ${ }^{3}$

\begin{tabular}{lcccc}
\hline MS , kg/año & ED, Mcal/año & N, kg/año & MS asignada $4 / a n ̃ o ~$ & EM, Mcal/año \\
334,15 & $1.154,896$ & 5,184 & 766,5 & 880,62 \\
\hline
\end{tabular}

ab Literales diferentes en el mismo renglón son diferentes $(\mathrm{P}<0,05)$.

1 ED: Energía digestible.

2 EM: Energía metabolizable.

3 Considerando un venado con un PV de $60 \mathrm{~kg}$, por lo que el peso metabólico ${ }^{3}$ (Peso vivo), ${ }^{75}$ es de 19,90 kg.

4 Es la cantidad de MS asignada de acuerdo a la presión de pastoreo. Kg de forraje por 3,5\% PV.

Cuadro 4. Capacidad de carga de venados de cola blanca (venados/ha) estimada por diferentes métodos y grupos vegetales. Carrying capacity of white-tailed deer (deer/ha) estimated using different methods and vegetal groups.

\begin{tabular}{|c|c|c|c|c|c|c|}
\hline \multicolumn{7}{|c|}{ Método de estimación } \\
\hline \multicolumn{7}{|c|}{ Disponibilidad de compuestos nutritivos } \\
\hline $\mathrm{MS}^{1}$ & Nitrógeno & $\mathrm{ED}^{2}$ & Presión de pastoreo & Metabolismo ecológico & & $\mathrm{EM}$ \\
\hline $1,043^{\mathrm{a}}$ & $0,604^{\mathrm{ab}}$ & $0,451^{b}$ & $0,387^{\mathrm{b}}$ & $0,256^{\mathrm{b}}$ & & 118 \\
\hline \multicolumn{7}{|c|}{ Grupo vegetal } \\
\hline Pastos & Herbáceas & Arbóreas & Arbustivas & Total & & $\mathrm{EM}$ \\
\hline $0,707^{\mathrm{b}}$ & $0,325^{\text {bc }}$ & $0,228^{c}$ & $0,033^{\mathrm{c}}$ & $1,449^{\mathrm{a}}$ & & 118 \\
\hline \multicolumn{7}{|c|}{ Densidad animal, venados /ha } \\
\hline Autor, (año) & 1975 & 1978 & 1984 & 1991 & 1996 & 2000 \\
\hline Romo, (1987) & & & $0,02-0,04$ & & & \\
\hline Villalobos, (1998) & 0,0065 & 0,0159 & 0,0241 & 0,0181 & 0,027 & \\
\hline Kobelkowsky y col (2000) & & & & & & 0,0231 \\
\hline
\end{tabular}


(DP) del venado cola blanca en los años del muestreo y subsecuentes publicados por Romo (1987), Villalobos (1998) y Kobelkowsky y col (2000). Dentro de métodos, la estimación de $K$ utilizando la MS generó el valor más alto, mientras que la estimación por PC produjo valores intermedios y el uso de ED, PP y EM generaron los valores más bajos. Dentro de grupos vegetales, la estimación más alta de $K$ se obtuvo con la sumatoria de la biomasa de los diferentes grupos, mientras que las gramíneas y las herbáceas generaron valores intermedios y tanto las arbóreas como las arbustivas tuvieron los valores menores; $\sin$ embargo, sólo estas últimas tuvieron un valor de $K$ menor a la DP. El análisis de los contrastes mostró que dentro de los métodos evaluados tanto la suma de las herbáceas con las arbustivas versus los pastos y las arbóreas como la de las arbustivas con las arbóreas versus los pastos y las herbáceas tuvieron una menor $K(0,15$ vs. $0,33 \mathrm{P}<0,01$ y 0,10 vs. $0,38 \mathrm{P}<0,002)$.

\section{DISCUSIÓN}

Las diferencias en la biomasa vegetal y en la cantidad de componentes nutritivos disponibles aportados por cada grupo vegetal pueden ser explicadas parcialmente en base a la ausencia de actividad ganadera que tenía el rancho antes de convertirse en coto de caza. Holecheck y col (2006) han revisado y discutido en forma abundante los efectos del pastoreo en la vegetación; sus datos muestran que dicha actividad incrementa la cantidad de gramíneas y reduce el número de arbustivas presentes en un sitio. Sin embargo, cuando dicha actividad desaparece por efecto de exclusión del ganado la sustitución de gramíneas por herbáceas y arbustivas es mínima si no se realiza una práctica cultural que estimule el crecimiento de las mismas (Adler y col 2004). También, trabajos publicados por Bates (2005) y Bates y col (2005) muestran que la producción de herbáceas y arbustivas se deprime por la ausencia de prácticas de corte o pastoreo de tal forma que se ocasiona una reducción en la biomasa de las mismas después de 4 años. Tobler y col (2003) mostraron que el pastoreo de bovinos modifica la proporción de especies gramíneas en un clima de sabana, por lo que bajo las condiciones de este trabajo, no es sorprendente que la biomasa aportada por los pastos sea mayor que la aportada por los otros grupos vegetales.

Por otro lado, la ausencia del efecto de época del año ha sido similar a la de otros estudios, que muestran que bajo un estado de equilibrio, la biomasa producida en una comunidad vegetal permanece relativamente constante a lo largo de las diferentes épocas del año, lo cual se explica como el resultado del incremento de la MS de algunas especies vegetales cuando otras tienden a disminuir su producción en el mismo momento (Buttolph y Coppock 2004).

La estimación de la capacidad de carga es un problema que ha sido tratado de resolver desde hace mucho tiempo utilizando diversas metodologías que incluyen la disponibilidad de la MS (McCall y col 1999), del hábitat de la zona (Warren y Krysl 1983), así como la concentración de proteína y ED de la dieta (McCall y col 1997), usando como referencia el requerimiento de MS, proteína o energía de un venado cola blanca estándar. Sin embargo, dentro de la literatura revisada, sólo el estudio de Mandujano (2007) ha contrastado la capacidad de carga estimada con la densidad poblacional, lo cual sería el criterio de comparación más cercano para la evaluación del parámetro estimado.

La comparación de la $K$ con la DP es importante, dado que de acuerdo con Mandujano (2007), cuando un área no tiene disturbios, la $K$ es igual a la DP más los animales muertos por los predadores y, en consecuencia, las $K$ estimadas correctamente son aquellas que se acercan más a la DP. En ese sentido, nuestros resultados indican que tanto los métodos como los componentes nutritivos seleccionados para determinar el requerimiento del animal tienen un impacto en la estimación de la capacidad de carga. A menor exigencia en el requerimiento de forraje o de nutrientes, mayor $K$ estimada. Por ejemplo, si se compara la capacidad de carga estimada a partir de la concentración de ED de los grupos vegetales $(0,451)$ con la estimada en base al metabolismo ecológico $(0,256)$, se puede apreciar que, al no considerar el gasto energético adicional al metabolismo basal lleva a una sobrestimación de la misma. El cálculo original de Moen (1978) para estimar el metabolismo ecológico del venado es laborioso; sin embargo, el uso de programas disponibles en plataformas permite determinar el requerimiento en forma sencilla considerando otros factores (época del año, sexo, estado fisiológico) y mejora la estimación del requerimiento energético evitando la subestimación del mismo. La metodología basada en presión de pastoreo tiene la ventaja de ser más sencilla en su uso para los manejadores de fauna silvestre, pues no requiere el cálculo del requerimiento energético del venado en vida libre.

Los resultados de esta estimación muestran que el uso de biomasa total, así como el de gramíneas, arbóreas y herbáceas, resulta en valores más altos que la DP, lo cual sobrestima el número de animales que puede soportar el área; en contraste, la estimación de $K$ con arbustivas resulta en valores que indican que podría estar cercana a la DP $(0,04$ venados/ha), dicha DP se mantiene a lo largo del tiempo durante al menos 15 años después del estudio (cuadro 2), lo que corrobora entonces que la estimación de capacidad de carga a partir de las arbustivas es un método que permite obtener valores cercanos a la misma. Aunque diversos investigadores han mostrado que el venado cola blanca tiene una dieta basada predominantemente en especies arbustivas y arbóreas (85\%), las herbáceas y gramíneas forman parte de su dieta (Gallina 1993, Plata y col 2009). También se ha demostrado la tendencia de dicha especie a migrar a áreas con una menor biomasa vegetal si esta es de mayor calidad nutritiva (Mandujano y col 2004). 
La estimación de la $K$ a partir de la sumatoria de todas las especies vegetales está fundamentada en las preferencias de los rumiantes por las especies vegetales y el hecho de que los venados tienen una dieta diversa, por lo que se puede utilizar la sumatoria de la biomasa de los diversos grupos vegetales que el animal consume (Stuth y Sheffield 2001). Sin embargo, nuestros resultados sugieren que el uso de biomasa total no es viable para estimar la $K$ porque la proporción de grupos vegetales consumidos no es homogénea, por lo que se requeriría identificar la preferencia del grupo vegetativo consumido por cada tipo de rumiante y así corregir los aportes.

La estimación de $K$ con base a las gramíneas resulta en valores mayores que la DP; además, su uso como estimador para venados puede ser biológicamente cuestionado. Estudios de composición de dieta del venado cola blanca en México indican que los pastos pueden estar del 0 al $6 \%$ (Martínez y col 1997, Clemente y col 2005, Plata y col 2009). La baja capacidad de los selectores de concentrados para incluir gramíneas en su dieta $(<25 \%)$ ha sido establecida claramente por Janis y col (2000) y está asociada al tamaño y requerimiento metabólico del animal, pues se ha establecido que las especies con menor tamaño tienen un mayor gasto energético y una mayor velocidad de tránsito intestinal que las especies grandes, por lo que están obligados a buscar tanto las especies vegetales como las partes más nutritivas de la planta que garanticen su supervivencia (McNaughton y Georgiadis 1986). La estimación de $K$ basada en herbáceas y arbóreas produce valores mayores a la DP. En el caso de las herbáceas se ha detectado que bajo condiciones de pastoreo tanto de animales domésticos como silvestres uno de los impactos más comunes es la modificación de la composición de tal forma que aumenta el número de plantas indeseables por dichas especies (Gonzales y Clements 2010), esta proliferación de especies no consumidas puede explicar el aumento de $K$ y la baja DP del sitio.

Por su parte, el uso de arbustivas estimaría una capacidad de carga muy similar a la DP, lo cual se puede explicar por el hecho de que este grupo constituye uno de los principales alimentos consumidos por el venado. En la zona de estudio, este grupo se ha encontrado como componente de la dieta del venado cola blanca desde el 39\% (Kobelkowsky 2000) hasta el 49\% (Clemente y col 2005). También, los trabajos de Stewart y col (2000) muestran la predilección que tienen los venados hacia los arbustos. Sus datos muestran que independientemente de la cobertura los venados prefieren áreas con más presencia de arbustivas que de herbáceas. Paton y col (1999) utilizaron la EM de este grupo vegetal para estimar la $K$ en áreas naturales de España con buenos resultados, por lo que en términos prácticos lo más sencillo sería estimar la $K$ a partir de la disponibilidad de MS de arbustivas utilizando el modelo con la presión de pastoreo, mientras que lo más complicado es estimar dicha variable a partir de la EM y el metabolismo ecológico del venado.
Se concluye que la determinación de la capacidad de carga en venado cola blanca debe considerar sus preferencias alimenticias, la selección del método y el impacto que tienen los requerimientos de nutrientes en la estimación del parámetro. Los métodos en base a energía y PP producen mejores estimaciones que los métodos basados en $\mathrm{N}$ o MS, dado que los resultados son más cercanos a la densidad poblacional y se esperaría que no pusiesen en riesgo a largo plazo el uso sustentable del recurso vegetal. Tanto el uso de biomasa total como de pastos, herbáceas y arbóreas producen una sobrestimación de la capacidad de carga, por lo no se recomiendan para estimar $K$. Bajo las condiciones de este trabajo la estimación de $K$ a partir de la biomasa disponible de las arbustivas produce la mejor aproximación a la DP del área.

\section{RESUMEN}

Debido a la importancia que tiene la metodología en la estimación de la capacidad de carga ( $K$ animales/ha) en venado cola blanca, los objetivos de este trabajo fueron: 1) Comparar la estimación de la $K$ por cinco métodos; tres basados en disponibilidad de compuestos nutritivos (materia seca (MS), energía digestible (ED) y nitrógeno (N)), otro basado en la presión de pastoreo y un último en el requerimiento de energía metabolizable (EM) estimado con base al metabolismo ecológico del venado, y 2) Comparar la $K$ estimada al usar la biomasa total o por los grupos de vegetales consumidos por el venado (gramíneas, herbáceas, arbustivas y arbóreas). Se utilizó la información de composición de la dieta y de biomasa de un rancho cinegético localizado en Aguascalientes, México. Los resultados fueron analizados de acuerdo a un modelo con arreglo factorial ( 5 x 5), donde los factores fueron el método de estimación y el grupo vegetal. Los valores de $K$ estimados con biomasa total fueron los mayores $(1,449)$, seguidos por los pastos $(0,707)$, luego por las herbáceas $(0,325)$, mientras que los estimados con arbóreas y arbustivas dieron los menores valores $(0,228$ y 0,03$)$. La estimación de $K$ por medio de plantas arbustivas $(0,033$ venados/ha) proporciona un valor más similar al de la densidad poblacional (0,02-0,04 venados/ha) que el estimado con otros grupos vegetativos, independientemente del método seleccionado (disponibilidad de MS, ED, N, metabolismo ecológico y la presión de pastoreo). Considerando la densidad poblacional $(0,02-0,04$ venados/ha) como el indicador apropiado para evaluar la capacidad de carga, se concluye que debe estimarse con base a la disponibilidad de arbustivas.

\section{REFERENCIAS}

Adler PB, DG Milchunas, WK Lauenroth, OE Sala, IC Burke. 2004. Functional traits of graminoids in semi-arid steppes: a test of grazing histories. J Appl Ecol 41, 653-663.

AOAC, Association of Official Analytical Chemists. 1995. Methods of Analysis. Washington D.C., USA.

Augustine DJ, PA Jordan. 1998. Predictors of white-tailed deer grazing intensity in fragmented deciduous forests. J Wild Manage 62, 1076-1085.

Bates JD. 2005. Herbaceous response to cattle grazing following Juniper cutting in Oregon. Rangeland Ecol Manag 58, 225-233.

Bates JD, RF Miller, T Svejcar. 2005. Long-term successional trends following western Juniper cutting. Rangeland Ecol Manage 58, 533-541.

Beauchamp VB, JC Stromberg. 2008. Changes to herbaceous plant communities on a regulated desert river. River Res Applic 24, 754-770. 
Beck JL, JM Peek, EK Strand. 2006. Estimates of elk summer range nutritional carrying capacity constrained by probabilities of habitat selection. J Wild Manage 70, 283-294.

Bryant FC, MM Kothmann, LB Merrill. 1979. Diets of sheep, angora goats, spanish goats and white-tailed deer under excellent range conditions. J Range Manage 32, 412-417.

Bullock J. 1999. Plants. In: Sutherland WJ (ed). Ecological census techniques. A Handbook. Cambridge University Press, Cambridge, UK.

Buttolph LP, DL Coppock. 2004. Influence of deferred grazing on vegetation dynamics and livestock productivity in an Andean pastoral system. J Applied Ecol 41, 664-674.

Clemente SF. 1984. Utilización de la vegetación nativa del venado cola blanca (Odocoileus virginianus). Tesis de Maestría, Colegio de Postgraduados, Montecillo, Estado de México, México.

Clemente SF, E Riquelme, GD Mendoza, R Barcena, S González, $R$ Ricalde. 2005. Digestibility of forage diets of white-tailed deer (Odocoileus virginianus, Hays) using different ruminal fluid inocula. J Appl Anim Res 27, 71-76.

Galbraith JK, GW Mathison, RJ Hudson, TA McAllister, KJ Cheng. 1998. Intake, digestibility, methane and heat production in bison, wapiti and white-tailed deer. Can J Anim Sci 78, 681-691.

Gallina SA. 1990. El venado cola blanca y su hábitat en la Michilia, Durango. Tesis Doctoral, Facultad de Ciencias, Universidad Nacional Autónoma de México, México.

Galt D, F Molinar, J Navarro, J Joseph, J Holechek. 2000. Grazing capacity and stocking rate. Rangelands 22, 7-11.

García E. 1981. Modificaciones al sistema de clasificación climática de Köppen para adaptarlo a las condiciones de la República Mexicana. Instituto de Geografía, Universidad Nacional Autónoma de México, México, D.F., México, Pp 145-150.

Gonzales EK, DR Clements. 2010. Plant Community Biomass shifts in response to mowing and fencing in invaded oak meadows with non-native grasses and abundant ungulates. Restoration Ecology $18,753-761$.

González CL, JH Everitt. 1982. Nutrient contents of major food plants eaten by cattle in the south Texas plains. J Range Manage 35, 733-736.

Gotelli NJ. 2008. Logistic population growth. In: Gotelli NJ (ed). A primer of ecology. Sinauer Associates Inc. Sunderland, Ma, USA, Pp 25-48.

Hansen RM, RC Clark, W Lawhorn. 1977. Foods of wild horses, deer, and cattle in the Douglas mountain area, Colorado. J Range Manage 30, 116-118.

Herrera JG, AB Serrano. 2005. Comparación de medias de tratamientos En: Martínez JA (ed). Análisis estadístico de experimentos agropecuarios. Colegio de Postgraduados, México, Pp 124-136.

Hobbs NT, DM Swift. 1985. Estimates of habitat carrying capacity incorporating explicit nutritional constraints. J Wild Manage 49, 814-822.

Holechek JL, TT Baker, JC Boren, D Galt. 2006. Grazing impacts on rangeland vegetation: what we have learned. Livestock grazing at light-to-moderate intensities can have positive impacts on rangeland vegetation in arid-to-semiarid areas. Rangelands 28, 7-13.

Jacoby PW. 1989. A glossary of terms used in range management: A definition of terms commonly used in range management. Society for Range Management, Denver, Colorado, USA, Pp 1-20.

Janis CM, J Damuth, JM Theodor. 2000. Miocene ungulates and terrestrial primary productivity: Where have all the browsers gone? PNAS 97, 7899-7904.

Jordan JS. 1967. Deer browsing in northern hard-woods after clear cutting. US Forest Serv Res Paper $\mathrm{N}^{\circ} 57$.

Kobelkowsky SR. 2000. Evaluación de hábitat y estructura de la población de venado cola blanca (Odocoileus virginianus) en la región central de la Sierra Fría, Aguascalientes. Tesis de Maestría, Colegio de Postgraduados, Montecillo, Estado de México, México.

Kobelkowsky-Sosa R, J Palacio-Núñez, F Clemente-Sánchez, GD Mendoza-Martínez, JG Herrera-Haro, J Gallegos-Sánchez. 2000.
Calidad del hábitat y estado poblacional del venado cola blanca (Odocoileus virginianus, Hays) en ranchos cinegéticos de la sierra fría, Aguascalientes. Revista Chapingo Serie Ciencias Forestales y del Ambiente 6, 125-130.

Mandujano S, S Gallina, G Arceo, A Pérez-Jiménez. 2004. Variación estacional del uso y preferencia de los tipos vegetacionales por el venado cola blanca en un bosque tropical de Jalisco. Acta Zoológica Mexicana (N.S.) 20, 45-67.

Mandujano S. 2007. Carrying capacity and potential production of ungulates for human use in a Mexican tropical dry forest. Biotropica $39,519-524$.

Martínez MA, V Molina, F González, S Marroquín, J Navar. 1997. Observations of white-tailed deer and cattle diets in México. J Range Manage 50, 253-257.

McCall DG, DA Clark, LJ Stachurski, JW Penno, AM Bryant, BJ Ridler. 1999. Optimized dairy grazing systems in the northeast United States and New Zealand. I. Model description and evaluation. J Dairy Sci 82, 1795-1807.

McCall TC, RD Brown, CL Bender. 1997. Comparison of techniques for determining the nutritional carrying capacity for white-tailed deer. J Range Manage 50, 33-38.

McLeod SR. 1997. Is the concept of carrying capacity useful in variable environments? Oikos 79, 529-542.

McNaughton SJ, NJ Georgiadis. 1986. Ecology of African grazing and browsing mammals. Ann Rev Ecol Syst 17, 39-65.

Miller KV, JM Wentworth. 2000. Carrying capacity. In: Demarais S, Krausman PR (eds). Ecology and management of large mammals in North America. Prentice Hall, Upper Saddle River, New Jersey, USA, Pp 140-155.

Moen AN. 1978. Seasonal changes in heart rates, activity, metabolism, and forage intake of white- tailed deer. $J$ Wild Manage 42, 715-738.

Otto R, JM Fernández-Palacios, BO Krüsi. 2001. Variation in species composition and vegetation structure of succulent scrub on Tenerife in relation to environmental variation. J Veg Sci 12, 237-248.

Paladines O, CE Lascano. 1983. Recomendaciones para evaluar germoplasma bajo pastoreo. En: Lascano CE (ed). Germoplasma forrajero bajo pastoreo en pequeñas parcelas, Vol. 1. CIAT, Colombia, Pp 165-183.

Plata FX, S Ebergeny, JL Resendiz, O Villarreal, R Bárcena, JA Viccone, GD Mendoza. 2009. Palatabilidad y composición química de alimentos consumidos en cautiverio por el venado cola blanca de Yucatán (Odocoileus virginianus yucatanensis). Arch Med Vet 41, 123-129.

Paton D, J Núñez-Trujillo, MA Díaz, A Muñoz.1999. Assessment of browsing biomass, nutritive value and carrying capacity of shrublands for red deer (Cervus elaphus L.) management in Monfragüe natural park (SW Spain) J Arid Environ 42, 137-147.

Robbins CT. 1973. The biological basis for the determination of carrying capacity. Doctoral dissertation, Cornell University, Ithaca, NY, USA.

Rogers O, TE Fulbright, DC Ruthven III. 2004. Vegetation and deer response to mechanical shrub clearing and burning. $J$ Range Manage 57, 41-48.

Romo DM. 1987. Dinámica de la población del venado cola blanca (O. virginianus) en la sierra de San Blas de pabellón del estado de Aguascalientes, México. Tesis de Biología, Centro Básico. Universidad Autónoma de Aguascalientes, México.

Sauvé DG, SD Côté. 2007. Winter forage selection in white-tailed deer at high density: Balsam Fir is the best of a bad choice. $J$ Wild Manage 71, 911-914.

Steel RG, JH Torrie. 1980. Principles and procedures of statistics: A biometrical approach. $2^{\text {nd }}$ ed. McGraw-Hill Book Company, New York, USA, Pp 172-233.

Stuth JW, AH Winward. 1977. Livestock-deer relations in the Lodgepole pine-pumice region of central Oregon. J Range Manage 30, 110-116.

Stuth JW, WJ Sheffield. 2001. Determining carrying capacity for combinations of livestock, white-tailed deer and exotic ungulates. In: 
Wildlife Management Handbook, Texas A\&M University System, Texas, USA, Pp 5-12.

Tobler MW, R Cochard, PJ Edwards. 2003. The impact of cattle ranching on large-scale vegetation patterns in a coastal savanna in Tanzania. $J$ Applied Ecol 40, 430-444.

Ullrey DE, WG Youatt, HE Johnson, LD Fay, BL Schoepke, WT Magee. 1970. Digestible and metabolizable energy requirements for winter maintenance of Michigan white-tailed does. J Wild Manage 34, 863-869.
Villalobos SV. 1998. El venado cola blanca en la sierra fría de Aguascalientes. Agric Rec Nat 2, 2-33.

Warren RJ, LJ Krysl. 1983. White-tailed deer food habits and nutritional status as affected by grazing and deer-harvest management. J Range Manage 36, 104-109.

Young CC. 1998. Defining the range: The development of carrying capacity in management practice. J History Biol 31, 61-83. 